Relations industrielles

Industrial Relations

\title{
Labor in a Free Society, edited by Michael Harrington and Paul Jacobs. University of California Press. Berkeley and Los Angeles, 1960. 186 pp. \$3.00.
}

\section{Gérard Dion}

Volume 16, numéro 1, janvier 1961

URI : https://id.erudit.org/iderudit/1021902ar

DOI : https://doi.org/10.7202/1021902ar

Aller au sommaire du numéro

\section{Éditeur(s)}

Département des relations industrielles de l’Université Laval

\section{ISSN}

0034-379X (imprimé)

1703-8138 (numérique)

Découvrir la revue

Citer ce compte rendu

Dion, G. (1961). Compte rendu de [Labor in a Free Society, edited by Michael Harrington and Paul Jacobs. University of California Press. Berkeley and Los Angeles, 1960. 186 pp. \$3.00.] Relations industrielles / Industrial Relations, 16(1), 130-131. https://doi.org/10.7202/1021902ar

Tous droits réservés @ Département des relations industrielles de l’Université Laval, 1961
Ce document est protégé par la loi sur le droit d'auteur. L’utilisation des services d'Érudit (y compris la reproduction) est assujettie à sa politique d'utilisation que vous pouvez consulter en ligne.

https://apropos.erudit.org/fr/usagers/politique-dutilisation/ 
(lois ouvrières et lois sociales) qui a permis une évolution ordonnée.

Devant cet échec de prédiction de la théorie de Marx, faut-il la rejeter complètement? En particulier, faut-il considérer que le conflit n'a plus de place dans la société industrielle post-capitalisme? L'auteur croit plutôt que la notion de conflit de groupe doit être conservée dans une théorie sociologique valable. Il reproche aux sociologues venus après Marx d'avoir rendu statique la notion de classe sociale. Le concept de classe est devenu l'équivalent de celui de strate sociale, et a servi surtout à décrire de façon statique la société.

On a ainsi perdu toute la richesse explicative contenue dans le concept marxiste de classe. Il est donc plus que jamais important de distinguer une théorie dynamique de la classe sociale, d'une théorie statique de la stratification sociale.

Dans la deuxième partie de son volume, l'auteur veut indiquer les principaux jalons dans l'établissement d'une théorie dynamique du changement social. A cette fin, il intente un procès à la plupart des théoriciens contemporains. Non seulement on a rendu statique le concept de classe, mais on a rendu statique toute la théorie (on a produit de la ( fausse monnaie $\gg)$. Dahrendorf distingue deux types fondamentaux de théorie sociologique. La théorie de l'intégration privilégie les éléments de cohésion dans la vie sociale. Le prototype de ce genre de théorie est la théorie structuro-fonctionnelle de Parsons. Ses postulats de base sont les suivants: 1) chaque société est une structure stable et relativement permanente; 2) chaque société est une structure bien intégrée, (en équilibre, tendant vers l'équilibre); 3) chaque élément de la structure a une fonction; 4) chaque structure sociale est basée sur un consensus dans le système de valeurs des membres. La théorie de la coercition privilégie les forces de contrainte et de résistance dans la vie sociale. Ses postulats affirment que 1) le changement social est toujours présent dans la vie de la société; 2) le conflit social est aussi toujours présent; 3) chaque élément de la société contribue à sa désintégration et à son changement; 4) chaque société est basée sur la domination d'une partie des membres sur les autres.

Une théorie sociologique véritable devrait tenir compte de ces deux types de théorie et en faire l'intégration. Cependant, à l'heure actuelle, ce sont les théories de l'intégration qui dominent la sociologie. C'est donc par réaction consciente que l'auteur veut élaborer les bases d'une théorie du conflit. Il cherchera à définir les notions de groupes d'intérêt, de groupes de conflits, de quasi-groupes et de changement social. Il faut retenir ici sa définition de la classe sociale comme un * groupe de conflit créé par la distribution différentielle de l'autorité dans les associations coordonnées de façon impérative. » Le centre de cette définition est la notion d'autorité (et non plus la richesse ou les biens de production comme chez Marx). Le concept de classe sociale peut donc ainsi devenir très utile pour analyser le changement social dans toute société industrielle qu'elle soit de type capitaliste, post-capitaliste, socialiste ou autre.

Par ses analyses critiques sur le plan théorique aussi bien que sur le plan de l'interprétation de l'évolution de nos sociétés industrielles, le volume de Dahrendorf apparaît donc comme une oeuvre dont l'importance sera sûrement très considérable dans le développement de la sociologie. Le fait que l'auteur suggère les problèmes plus qu'il ne les résoud, stimule le lecteur plus qu'il le frustre. Après la lecture du volume, on souhaite quand même que Dahrendorf produise bientôt un essai de théorie générale plus complet.

Gérald Fortin

Labor in a Free Society, edited by Michael Harrington and Paul Jacobs. University of California Press. Berkeley and Los Angeles, 1960. 186 pp. $\$ 3.00$.

The Fund for the Republic est une institution qui s'est donnée comme objectif d'approfondir les problèmes de la liberté humaine dans notre société industrielle et démocratique en évolution. Si tout le monde est d'accord pour constater le défi que présente à l'épanouissement de la liberté, le développement de l'industrialisation, on est loin de s'entendre sur les moyens à prendre pour trouver des solutions. La raison, c'est que l'on n'est pas encore arrivé à apprécier de la même façon des notions aussi importantes que celles de l'homme, de la liberté, de la société. Au fond, on est en face de divergences portant sur des valeurs que l'on présume communes, 
alors qu'elles ne le sont que superficiellement.

Une telle conclusion découle clairement des études que l'on peut lire dans cet ouvrage, études qui ont été présentées à un symposium organisé par The Fund for the Republic, en 1958. Huit personnes bien connues dans le monde des relations du travail ont apporté leur contribution et les éditeurs, dans un chapitre spécial ont tenté de résumer les discussions.

Erich Fromm, spécialiste en psychologie, étudie le problème de la liberté dans le milieu du travail. Sumner H. Slichter, réputé pour ses travaux sur le syndicalisme, analyse la position des syndicats ouvriers dans l'économie américaine. Archibald Cox montre le rôle que peut jouer la législation dans la sauvegarde de la démocratie syndicale. David Cole et Arthur J. Goldberg insistent davantage sur la capacité que possèdent les syndicats d'arriver à la même fin grâce à leurs propres moyens. Enfin, deux expériences étrangères sont décrites: celle de l'Angleterre par Hugh A. Clegg, et celle de l'Australie par James P. McClelland.

Bien que tous les auteurs poursuivent un même but et s'entendent sur la nécessité de sauvegarder la liberté des travailleurs, la démocratie syndicale, le bien de l'ensemble de la communauté, il n'est pas facile de réconcilier des vues aussi divergentes que celles de Slichter et celles de Goldberg. Par ailleurs tous les collaborateurs insistent sur l'erreur qu'il y a à identifier la démocratie syndicale avec la démocratie politique. De plus, l'expérience démontre que l'on doit tenir compte des facteurs environnants et qu'il n'existe pas de panacée: dans un contexte différent, des moyens identiques produisent des effets contraires.

Par la nature des choses, cet ouvrage ne nous présente pas un développement logique de la question centrale qui $y$ est abordée, les travailleurs dans une société libre ». Par lui-même, cependant, il est une expression de la démocratie en donnant au lecteur la possibilité de connaître un éventail d'opinions. Il a le mérite de faire voir la difficulté du problème et la nécessité de continuer à l'approfondir, car il est évident que le mouvement ouvrier américain entre dans une nouvelle phase de son existence et doit faire face à des situations qu'il n'a pas connues auparavant.

Géraro Dion
La situation syndicale aux Etats-Unis. Rapport d'une mission du Bureau international du travail. Genève. 1960. 202, rue Queen, Ottawa. 170 pp. $\$ 1.25$.

L'Organisation internationale du travail vise à établir des normes dans le champ des relations du travail. Grâce aux conventions, les pays membres sont amenés à les accepter et à les faire respecter. Toutefois, entre la ratification d'une convention, l'établissement de mesures législatives et l'application pratique, il peut exister un décalage considérable. Pour être effectives, il ne suffit pas que les garanties du droit d'association et d'action syndicale soient consacrées par un statut, il faut qu'elles se traduisent constamment dans les faits et que leur jouissance n'en soit pas restreinte.

Plusieurs méthodes ont été successivement tentées par l'O.I.T. dans le but de remplir son rôle de surveillance d'une façon satisfaisante. D'abord l'étude des plaintes relatives à la violation des conventions. Ensuite l'étude des documents et autres sources d'information de caractère officiel. La première méthode ne peut produire que des résultats limités et superficiels. La seconde ne donne pas une vue complète de la réalité. Voilà pourquoi en 1958, le Conseil d'administration a approuvé une résolution instituant un mécanisme permettant d'obtenir des renseignements non seulement sur la situation juridique, mais encore sur la situation véritable de chacun des Etats Membres. Des missions d'études iraient faire enquête sur place dans les différents pays. Or, les deux premiers gouvernements qui ont accepté d'autoriser cet organisme extérieur à enquêter sur leur territoire sont les Etats-Unis et l'U.R.S.S. En 1959, une mission s'est rendue dans chacun de ces pays. Le Bureau international du travail vient de publier dans deux ouvrages distincts le rapport de cette mission.

La situation syndicale aux Etats-Unis est une synthèse intéressante, même pour ceux qui connaissent la vie, le développement et l'action syndicale dans ce pays. Présentée par des observateurs étrangers qui manifestent un souci d'objectivité, cette synthèse permet de saisir mieux certains aspects que l'on peut ètre porté à négliger.

Ainsi, le syndicalisme américain, pos- 